\title{
Computer-Assisted Three-Dimensional Planning for Orbital Decompression
}

\author{
Nicholas Mahoney, MD ${ }^{2}$ Michael P. Grant, MD, $\mathrm{PhD}^{1,2} \quad$ Srinivas Murthy Susarla, MD, DMD, MPH \\ Shannath Merbs, MD, $\mathrm{PhD}^{2}$
}

${ }^{1}$ Department of Plastic and Reconstructive Surgery, Johns Hopkins Hospital, Baltimore, Maryland

2 Division of Oculoplastic Surgery, Department of Ophthalmology, Wilmen Eye Institute, Johns Hopkins Hospital, Baltimore, Maryland

Address for correspondence Srinivas Murthy Susarla, MD, DMD, MPH, Department of Plastic Surgery, Johns Hopkins Hospital, 801 N. Caroline Street, Baltimore, MD 21205 (e-mail: ssusarl1@jhmi.edu).

\begin{abstract}
Keywords

- orbital decompression

- three-dimensional planning

- orbital osteotomy

- Graves disease

- orbital reconstruction

Thyroid-associated orbitopathy is the most common cause of unilateral or bilateral proptosis in adults. A mainstay of surgical treatment is orbital decompression utilizing osteotomies to increase the size of the affected bony orbit to accommodate the larger soft tissue volume. Over the past several decades, numerous approaches have been described for orbital decompression. However, given the intricate osseous and soft tissue anatomy within the orbit, orbital decompression is a potentially hazardous intervention. With advances in three-dimensional imaging and virtual planning, extensive orbital decompressions can be performed safely and efficiently. In this report, we describe two cases of three-wall orbital decompressions using three-dimensional planning.
\end{abstract}

Thyroid-associated orbitopathy is the most common inflammatory disorder of the orbit and remains the most common cause of unilateral or bilateral proptosis in adults. ${ }^{1-3}$ Since being first described by Parry in the late 18th century and then by Graves in the early 19th century, extensive study has better elucidated the underlying pathophysiology and refined both the medical management and surgical approaches. ${ }^{4-12}$

Clinical features can be subtle in the early phases and highly variable, with misdiagnosis common due to the diversity of presentations. ${ }^{1-5}$ The disorder is more common in females than in males (4:1) and tends to be more severe in patients younger than 40 years or older than 60 years. ${ }^{2,3}$ While the clinical severity can become progressively worse in the 1.5 to 3 years following initial presentation, more than $60 \%$ of patients display some spontaneous improvement without intervention. ${ }^{2,3}$

Although the catalyst for thyroid-related orbitopathy has not been fully expounded, it is currently accepted that the inflammatory nidus is, in part, related to acquired thyroidstimulating hormone-receptor (TSH-R) autoimmunity. ${ }^{5-7} \mathrm{Ac}-$ tivation of TSH-R in orbital fibroblasts results in deposition of hyaluronic acid, deposition of GAG-proteins, and activation of

received

February 9, 2014

accepted after revision

April 21, 2014

published online

November 14, 2014 immune modulators leading to myofibroblast differentiation..$^{5-7}$ The end result is a proinflammatory state within the orbit, ultimately resulting in orbital fat hypertrophy, extraocular muscle enlargement, and tissue fibrosis. Clinically, this manifests as periorbital edema, proptosis (with or without corneal exposure), restrictive strabismus, eyelid retraction, and, occasionally, optic neuropathy. ${ }^{1,2,7}$

The resulting enlargement of the soft tissue content of the orbit relative to the fixed volume of the bony orbit leads to exophthalmos, which often requires surgical intervention(s) designed to enlarge the orbit and/or remove orbital fat. These approaches have been successfully utilized to treat globe malposition. ${ }^{8-18}$ Typically, this is performed once the disease state is considered inactive based on serial clinical examinations and monitoring of thyroid-stimulating immunoglobulin (TSI). Orbital decompression can alter axial and vertical globe position, rotation, alignment, and relative relationships to periocular soft tissue structures and thus precedes all other orbital surgical interventions (e.g., strabismus surgery, eyelid retraction repair, blepharoplasty). ${ }^{8}$

More than a dozen algorithms have been described for orbital decompression surgery, with variable proptosis
Copyright $\odot 2015$ by Thieme Medical Publishers, Inc., 333 Seventh Avenue, New York, NY 10001, USA. Tel: +1(212) 584-4662.
DOI http://dx.doi.org/ 10.1055/s-0034-1393731. ISSN $1943-3875$. 
reduction amounts and complication rates. ${ }^{8-18}$ Recent data suggest the transconjunctival and endoscopic approaches are associated with lower complication rates and better globe proptosis reduction when compared with transpalpebral and transantral approaches. ${ }^{9,10,12}$ One-, two-, three-, and fourwall decompressions have been described in the literature, though current data suggest that there is little need to decompress the orbital roof. ${ }^{9,10,12}$ The decision to proceed with one-, two-, or three-walled surgery should be individually tailored to the specific case. ${ }^{16}$

Though there is extensive literature describing the methods to expose the orbit and perform fat and bony decompression, there is little discussion on the use of computer-assisted techniques to aid in planning, intraoperative navigation, and postoperative quality control. ${ }^{16,18}$

This report describes the use of three-dimensional computed tomography (CT) for planning combined with intraoperative navigation for multiwalled orbital decompression in cases of asymmetric exophthalmos.

\section{Patient 1}

A 49-year-old African American male patient was referred to our clinic for evaluation and management of thyroid-related orbitopathy. Three years prior to presentation, the patient had presented to his primary care physician with worsening double vision, dry eyes, and photophobia. A diagnosis of Graves disease was established, and the patient underwent treatment with intravenous steroids for several months, followed by a total thyroidectomy. Vertical rectus muscle surgery had been attempted with minimal improvement. In addition, surgical correction of his left lower eyelid retraction was performed resulting in no significant change in position. Following these interventions, he remained bothered by persistent diplopia and was referred to our institution for evaluation and possible surgical decompression. At the time of presentation, he was maintained on a stable dose of thyroid hormone-replacement therapy and had a normal TSH level. His medical history was otherwise notable for hypertension managed pharmacologically.

Physical examination was notable for 20/15 corrected visual acuity bilaterally, full confrontational visual fields, and no color deficits. Extraocular movements were restricted in supraduction and abduction bilaterally, but greater on the left than the right. There was bilateral asymmetric exophthalmos (Hertel exophthalmometry measurements of $25 \mathrm{~mm}$ on the right and $28 \mathrm{~mm}$ on the left with a base of $124 \mathrm{~mm}$ ) as well as lower eyelid retraction with $2 \mathrm{~mm}$ of inferior scleral show on the right and $3 \mathrm{~mm}$ on the left (-Fig. 1). The ocular exam was notable for bilateral conjunctival hyperemia and mild corneal epithelial erosion on the left but was otherwise normal.

A helical, thin section orbital CT scan $(0.625 \mathrm{~mm}$ slice thickness) demonstrated exophthalmos with marked enlargement of the superior, medial, and inferior rectus muscles bilaterally.

\section{Preoperative Planning}

DICOM data from the CT study were imported into the planning software (iPlan CMF 3.0 BrainLab, Brainlab Inc., Westchester, IL) for preoperative planning. For each wall involved, the planned resection sites were indicated using different colors (-Fig. 2). A one-wall (orbital floor) decompression was planned on the right side and a three-wall decompression (lateral wall, floor, and medial wall) was planned for the left side.

When planning bony removal for the orbital floor, the infraorbital canal was located centrally and maintained, allowing preservation of the infraorbital nerve and preventing globe descent into the maxillary sinus.

The lateral wall was assessed with attention to the size and shape of the greater wing of the sphenoid bone. This patient had a slightly concave contour associated with a narrow posterior orbit, which was marked for removal. The posterior extent of the resection was designed to maximize the decompression and minimize the risk of a CSF leak.

The inferior boundary of the medial was the inferomedial strut, representing the superior portion of the frontomaxillary buttress, which was maintained to allow support to the central facial skeleton. The superior boundary was the frontoethmoidal suture line (i.e., a radiographic line between the anterior and posterior ethmoidal arteries) to allow for a safe zone of bony removal without encroaching upon the cribriform plate.

\section{Intraoperative Course}

Intraoperative navigation was initiated once the patient was placed under general anesthesia. The reference array was affixed to the supratemporal skull using a $4 \mathrm{~mm}$ screw avoiding the midline frontal bone to prevent entry into the
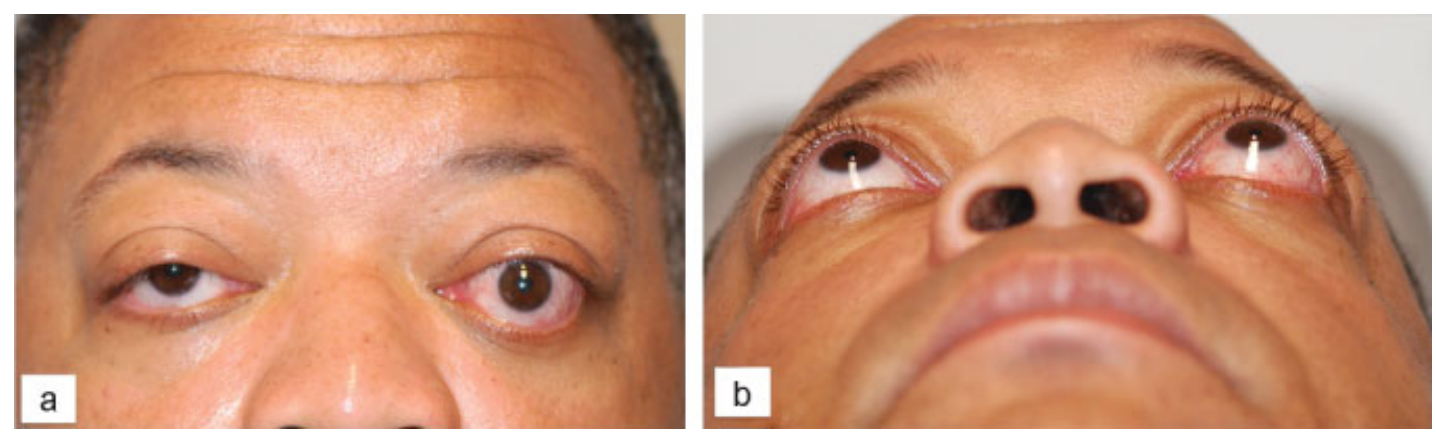

Fig. 1 Patient 1, frontal (panel A) and submentovertex (panel B) views of a 49-year-old man with exophthalmos related to thyroid eye disease before orbital decompression surgery. 


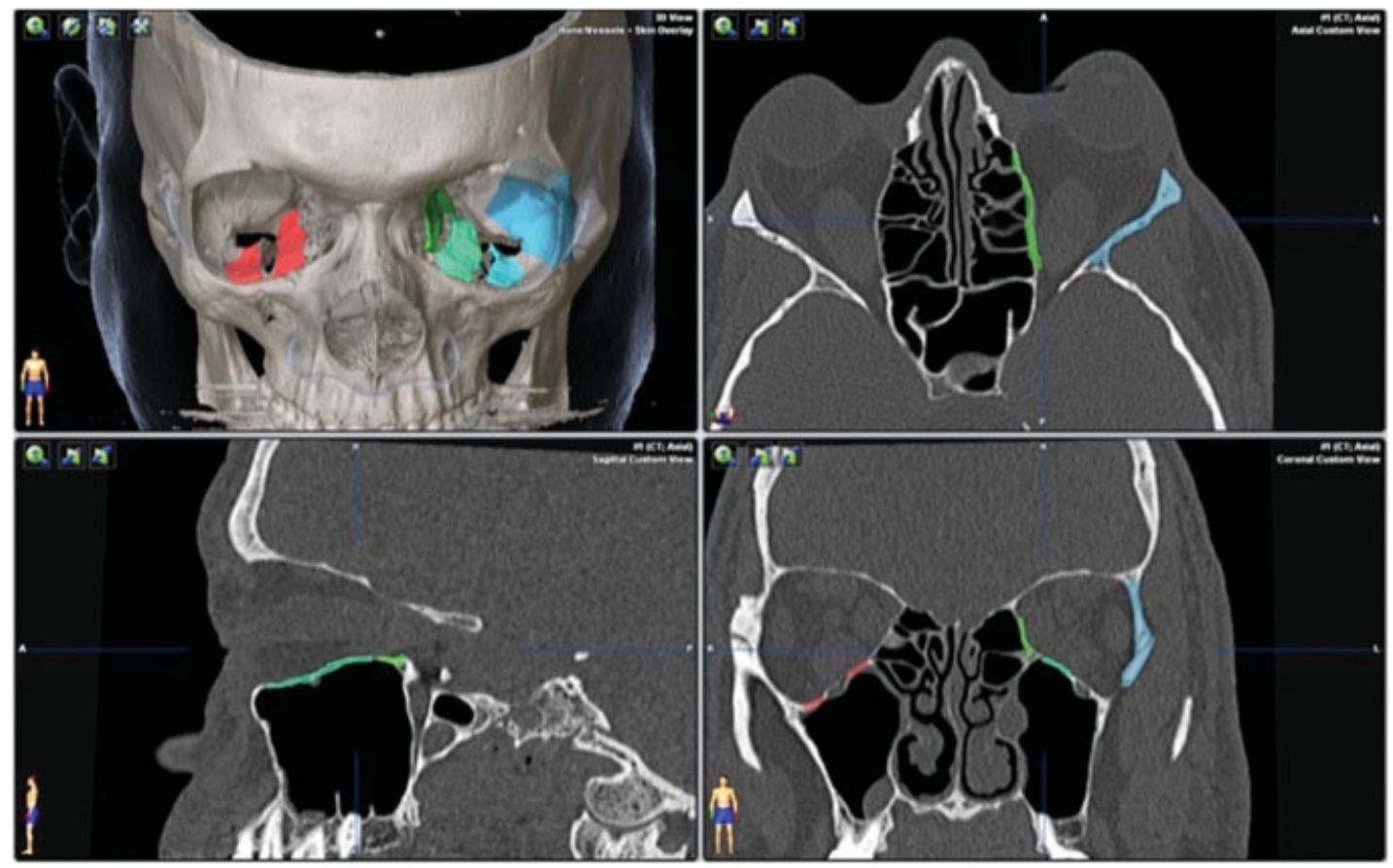

Fig. 2 Axial, coronal, and sagittal views with 3D reconstruction for patient 1, showing the preoperative plan. Areas of planned decompression are highlighted in color.

sagittal venous sinus. Soft tissue landmarks were mapped with an infrared light source to register the patient and array to the CT images. Intraoperative navigation was completed through the use of a frameless stereotaxy. Throughout the operative course, the surgeon verified the registration against bony landmarks such as at Whitnall tubercle on the lateral rim and the inferior edge of the nasal bone. We chose a bony reference point because, in our experience using both bony and soft tissue surface registration points around the orbit, our accuracy in the orbit is very high. There is no additional step needed, such as dental molds, and our preoperative scanning protocol does not need to radiate outside of the surgical field.

The lateral wall was approached via an incision at the lateral canthus using a \#15 blade, and a canthotomy was completed with Westcott scissors. The lateral orbital rim periosteum was identified, and the horizontal canthotomy incision was continued between the superior and inferior limbs of the lateral canthal tendon. A second perpendicular incision in the periosteum along the lateral rim allowed further exposure of the lateral orbital wall. Subperiosteal dissection was performed superiorly above the frontozygomatic suture and inferiorly to the zygomatic arch. Dissection was taken into the orbit to the inferior orbital fissure. The BrainLab pointer was then used to verify the positioning and plan for the lateral orbitotomy (-Fig. 3). A reciprocating saw was used to make horizontal osteotomies at the level of the orbital roof superiorly and the zygomatic arch inferiorly. A rongeur was then used to in-fracture the lateral orbital wall and rim. The temporalis was detached from the posterior lateral wall using Bovie electrocautery, and the bone fragment was removed. Finally, incisions were made in the periorbita parallel to the lateral rectus, to allow for soft tissue prolapse into the temporal fossa.

Next, the orbital floor and medial wall were approached via a retroseptal transconjunctival incision with a retrocaruncular
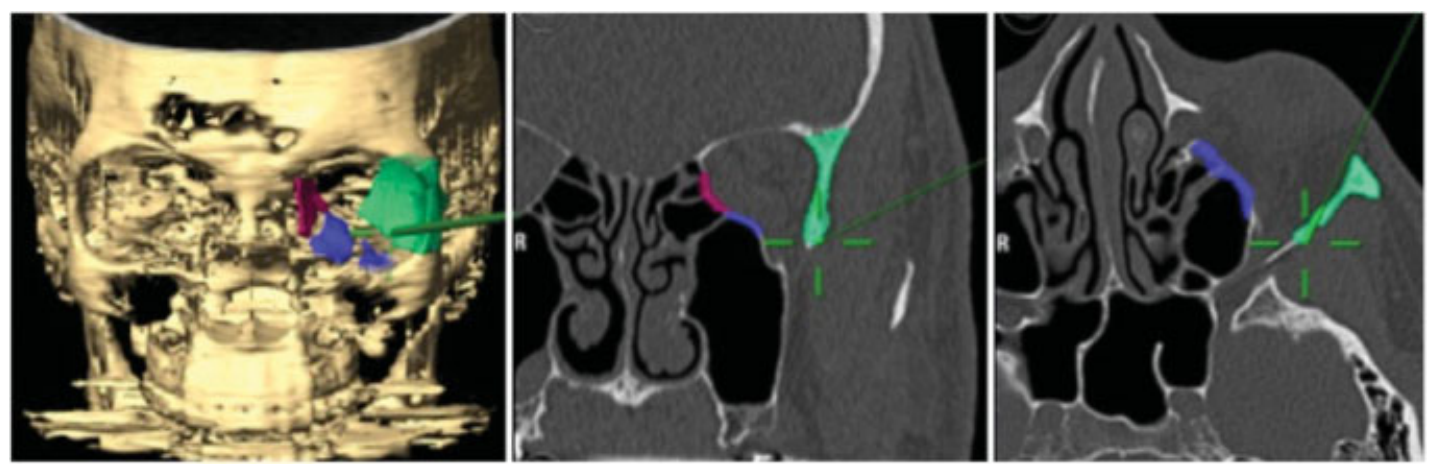

Fig. 3 Intraoperative navigation during lateral wall removal demonstrating the location of the pointer (green line with cross-hairs). 

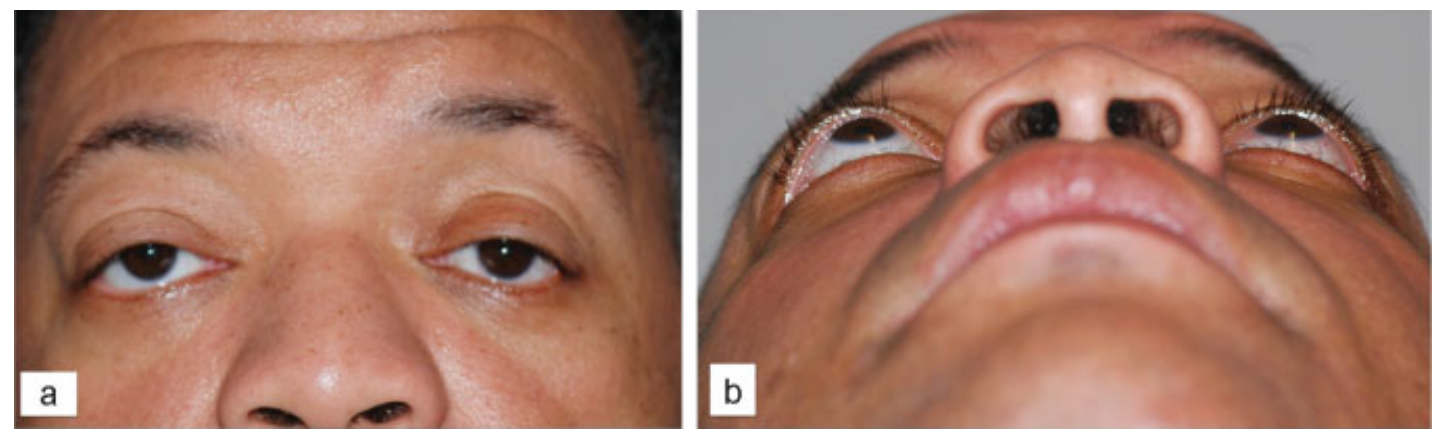

Fig. 4 Patient 1, frontal (panel a) and submentovertex (panel b) views after asymmetric orbital decompression surgery, upper and lower lid retraction repair, and lateral canthal surgery.

extension. ${ }^{19-21}$ Bovie electrocautery was used to make the inferior fornix incision, with the medial extension between the caruncle and plica. Dissection was continued to the orbital floor inside of the orbital rim preserving the arcus marginalis and mid-face suspensory ligaments. The extraconal fat was immediately encountered with this approach and a portion was resected to improve visibility. Along the medial wall, the periorbita was incised posterior to the posterior lacrimal crest and the Brainlab pointer was used to identify the anterior ethmoidal foramen. Once identified, this was used as the superior extent of the medial wall resection. Bone from the orbital floor was removed using small osteotomes and sphenoid punches, with care taken to preserve the infraorbital nerve. A Freer periosteal elevator and Webster needle holder were used to remove bone along the medial wall. The intraconal fat was accessed through the inferolateral quadrant of the muscle cone and gently debulked.

The lateral canthal angle was re-approximated using a 6-0 polyglactin 910 suture and the lateral canthal skin incision was closed using 8-0 nylon. Prednisolone-gentamycin ointment was placed in the eye. $\mathrm{A} 1 / 4$ " Penrose drain was placed in the transconjunctival incision for drainage and was secured with a 6-0 nylon Frost suture. The eye was then dressed with a tight patch displacing the orbital contents into the regions of bone removal.

In this manner, the left orbit was decompressed. The patient was admitted for overnight observation and discharged on postoperative day 1 . His patch was removed to confirm stable visual acuity and then replaced for 3 additional days. His infraorbital nerve sensation was noted to be intact at the first postoperative visit. He underwent a single-wall decompression of the right orbit in a similar fashion 2 weeks after the original operation. The contralateral side was also completed without complication.

\section{Postoperative Course}

A pressure patch was placed over the operative eye postoperatively and maintained for 3 days. The patient was discharged home in stable condition on postoperative day 1 . The pressure patch is utilized as a means of prolapsing the orbital contents into the temporal space (when discussing surgery with patients, we discuss the option with our patients and they typically elect sequential surgery to avoid being patched closed bilaterally for 3 days).
At the 3-month follow-up, he was noted to have dramatic improvement in his exophthalmos, as well as improvement in extraocular movements and diplopia. He subsequently underwent right lower eyelid retraction repair and lateral tarsorrhaphy, as well as left upper eyelid retraction repair, with improvement in dryness. He remained completely asymptomatic (no diplopia, restriction in movement, persistent dryness, or corneal irritation) at 10-month follow-up (-Fig. 4).

Three months after the decompression, the patient had a postoperative helical CT study. The postoperative imaging demonstrated the bony removal and decompression of the orbital soft tissues ( $\mathbf{F i g . ~ 5 )}$ ). Virtual stereolithographic models were generated from the DICOM data and used for direct comparison of the pre- and postoperative orbits ( - Fig. 6 ).

\section{Patient 2}

A 56-year-old Caucasian male patient with hyperthyroidism presented with progressively worsening double vision over a 4-year period. He also noted bilateral dry eyes and photophobia. He had no other significant medical history. He had been managed medically with propylthiouracil and had a normal TSH and stable serial TSI levels at the time of presentation.

Physical examination was notable for 20/25 visual acuity bilaterally, with full visual fields and no color deficits. Extraocular movements were restricted in supraduction and abduction bilaterally. There was bilateral but asymmetrical

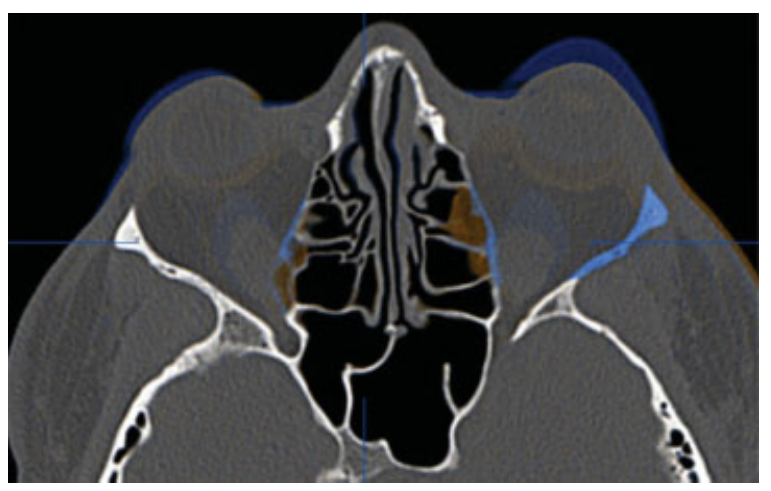

Fig. 5 Fusion of preoperative (blue) and postoperative (orange) CT scans for patient 1 . Uncolored portions are identical on the two scans. 

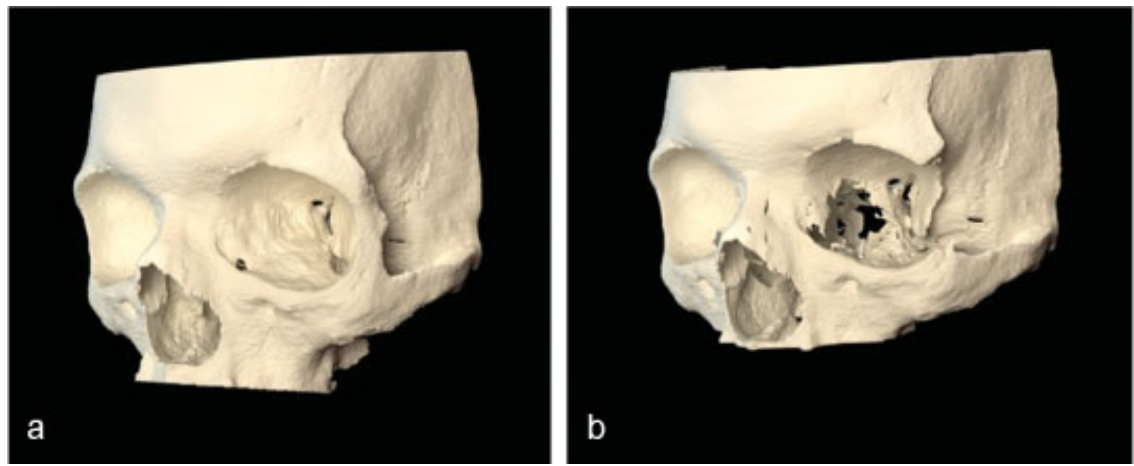

Fig. 6 Virtual stereolithographic rendering before (a) and after (b) decompression for patient 1.

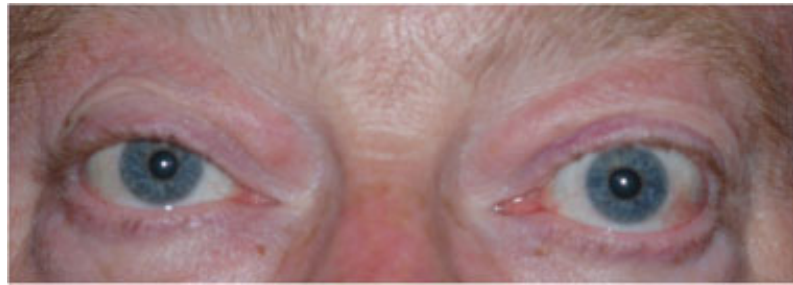

Fig. 7 Patient 2, a 57-year-old man with significant exophthalmos related to thyroid eye disease before orbital decompression surgery.

exophthalmos (Hertel exophthalmometry measurements of $25.5 \mathrm{~mm}$ on the right and $27 \mathrm{~mm}$ on the left with a base of $117 \mathrm{~mm}$ ) as well as eyelid retraction with inferior scleral show of $1 \mathrm{~mm}$ on the right and $2 \mathrm{~mm}$ on the left ( - Fig. 7). The ocular exam was notable for bilateral conjunctival hyperemia and mild cataracts, but was otherwise normal.

A helical, thin cut orbital CT scan $(0.625-\mathrm{mm}$ slice thickness) demonstrated exophthalmos with marked enlargement of the superior, medial, and inferior rectus muscles bilaterally.

Similar to the first case, DICOM data from the CT study were utilized to plan a two-wall (lateral wall and orbital floor) decompression on the right side and a three-wall decompression (lateral wall, floor, and medial wall) on the left side (-Fig. 8).

The decompression was performed using the same approach as detailed in the first case with the same postoperative regimen. There were no complications.

At the 3-month follow-up, he was noted to have dramatic improvement in his exophthalmos (-Fig. 9), as well as improved extraocular motility. He subsequently underwent strabismus surgery followed by left upper eyelid retraction repair, with improvement in dryness and resolution of diplopia. No complications were noted.

Three-month postoperative imaging demonstrated the bony removal and decompression of the orbital soft tissues ( - Fig. 10). Virtual stereolithographic ( - Fig. 11) and
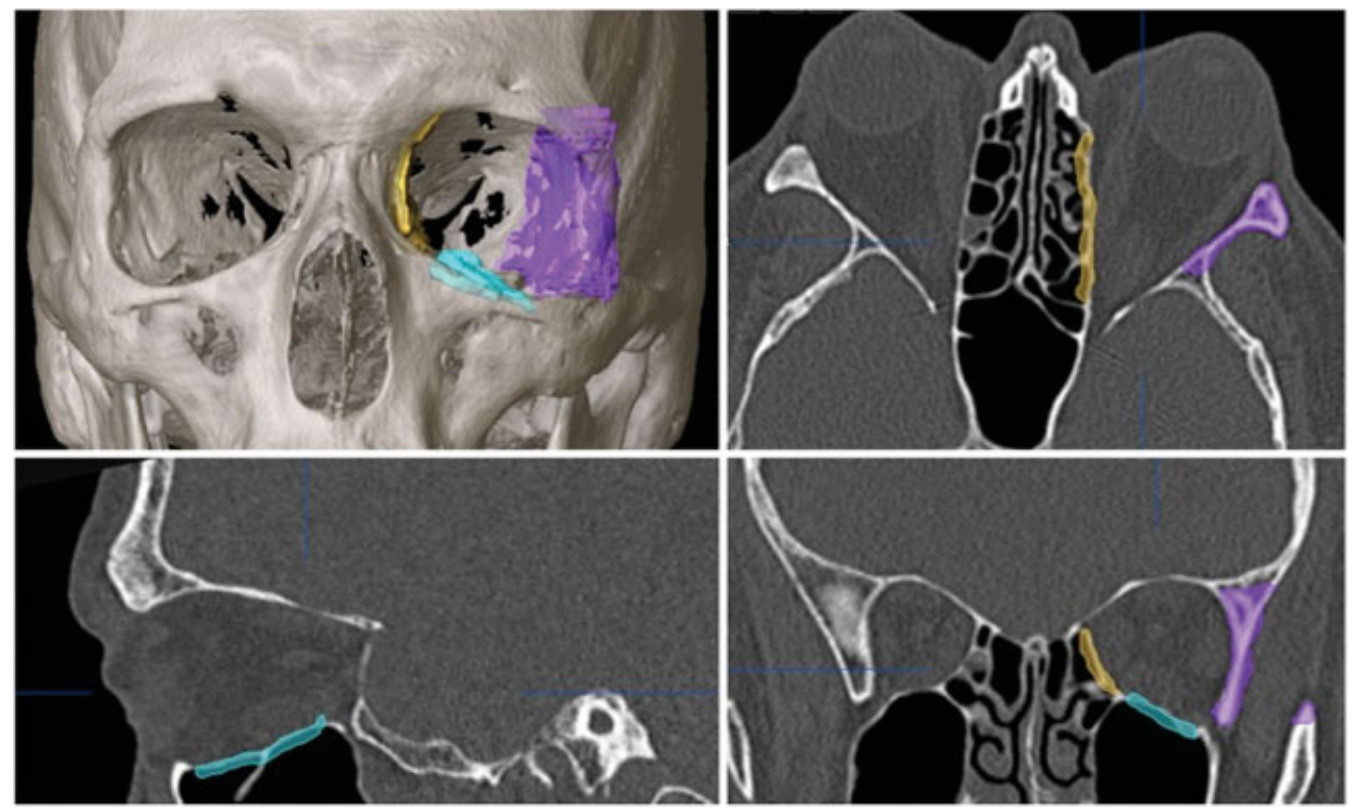

Fig. 8 Axial, coronal, and sagittal views with 3D reconstruction for patient 2, showing the preoperative plan for the left-sided, three-wall orbital decompression surgery. 


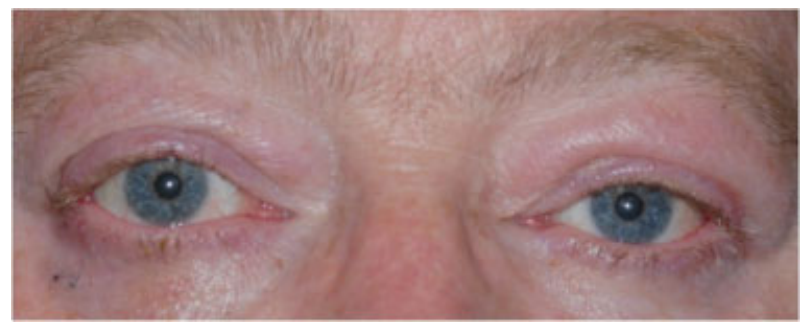

Fig. 9 Patient 2 after asymmetric orbital decompression surgery as well as eyelid correction and strabismus surgery.

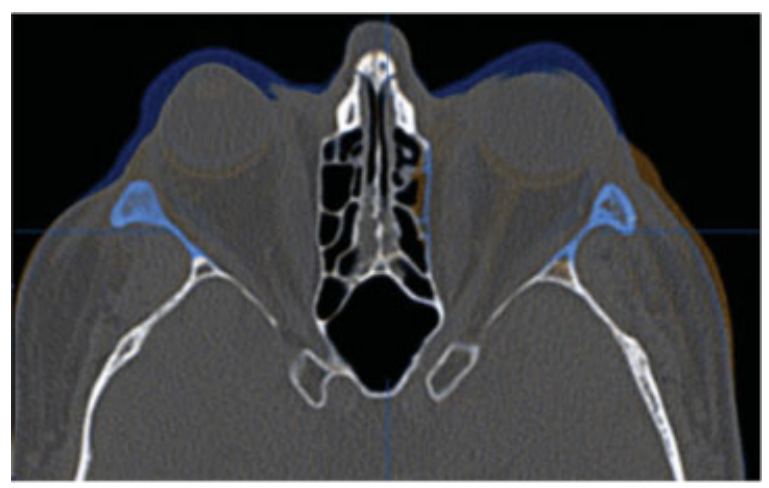

Fig. 10 Fusion of preoperative (blue) and postoperative (orange) CT scans for patient 2 . Uncolored portions are identical on the two scans.

three-dimensional volumetric models were generated from the DICOM data and used for direct comparison of the preand postoperative orbits.

\section{Discussion}

Surgical decompression of the orbit through various techniques is an established component of the surgical management of thyroid eye disease. ${ }^{8-18}$ The utility of the decompression is to correct the ratio of the soft tissue volume to the bony volume. As the extraocular muscles increase in size due to inflammation, the fixed volume of the bony orbit results in an unfavorable ratio of soft tissue to bone. As a result, extraocular muscle function is limited, diplopia may result, and proptosis is evident. Judicious expansion of the bony orbit rectifies this unfavorable volume relationship. As surgical decompression precedes and influences all other surgical corrections, it is necessary that it be done safely and effectively with a single procedure to facilitate the further rehabilitation of patient with thyroid eye disease. The challenging regional anatomy of the orbit, with several critical cranial nerves and vascular structures in regions that cannot always be clearly visualized, and the often asymmetric nature of the exophthalmos, is an ideal setting to use preoperative planning and intraoperative navigation. This report describes the use of computer-assisted stereotactic navigation for orbital decompression in cases requiring bilateral asymmetric decompression.

By using stereotactic navigation and the associated software platforms, the operator can preoperatively plan the resection, improve intraoperative safety and precision, and perform postoperative quality control to assess surgical accuracy. The technique is sufficiently versatile and is useful for different approaches (e.g., transconjunctival, transnasal) to the difficult anatomic regions (i.e., medial orbital wall). Preoperative targeted bone removal is particularly useful in enabling consistent and predictable decompressions. Pre-existing variation in orbital anatomy can be assessed and often influences the plan for targeted bone removal. The preoperative anatomical assessment and intraoperative use of navigation throughout the case promotes safety, particularly in avoiding optic nerve injury along the posterior medial wall and breach of the dura at the posterior lateral wall. Finally, preoperative planning followed by postoperative assessment of decompression surgery allows for the potential for an objective outcome assessment as our ability to quantify the magnitude of decompression improves.

Although there are upfront costs associated with obtaining the necessary materials for planning and the intraoperative navigation tools, such tools are readily available at most major academic centers, as they are routinely utilized by neurosurgery and otolaryngology, among other services. In addition, in our experience, increased efficiency in the operating room (up to $40-50 \%$ ) will translate to decreased operating and general anesthetic times, which will have a direct impact on overall cost.
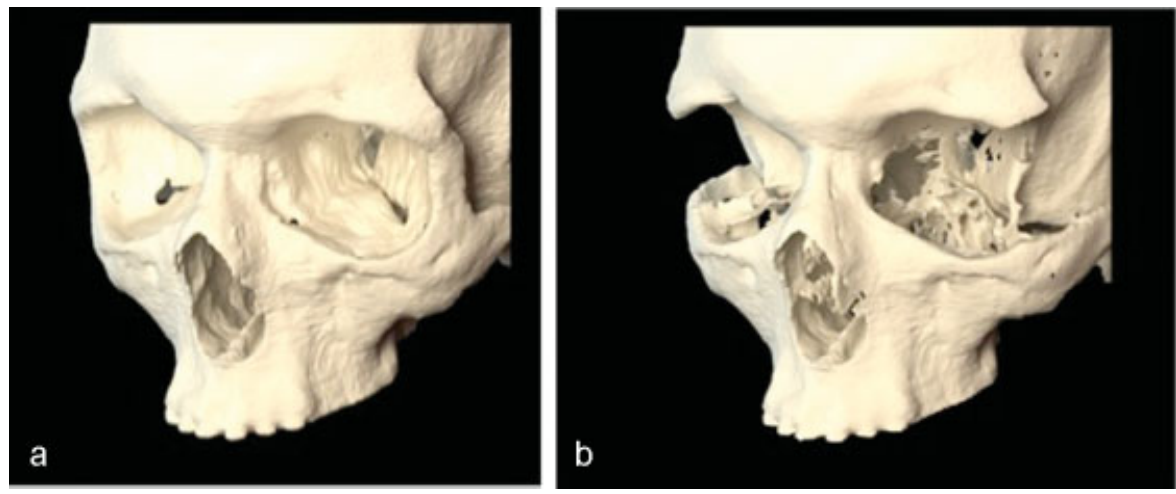

Fig. 11 Virtual stereolithographic rendering before (a) and after (b) decompression for patient 2. 
In the cases discussed herein, we routinely perform Hertel exophthalmometry as it is reproducible and easy to perform. After lateral rim removal, we clinically measure A-P globe position using the Naugle exophthalmometer; however, we do not have measurements with the Naugle preoperatively in these two cases. Rather, the reduction in proptosis in these two patients was easily verified on their pre- and postoperative CT scan fusion (-Figs. 5 and 10). Objective outcome assessment utilizing the position of the anterior cornea to the orbital superior (Os) and orbitale inferior (Oi) is ongoing and will be the goal of future works.

An additional shortcoming of our present approach is our limited ability, at the present time, to quantify fat decompression. Limited fat decompression was performed in both cases, but was not directly quantified. In addition, although anticipated as a portion of the procedure preoperatively, the preoperative planning does not explicitly include soft tissue decompression. We are beginning to measure the amount of fat resected intraoperatively and hope to develop a quantitative CT-based method for the planning and postoperative assessment of fat resection.

Of note, in these cases, the lateral orbital wall was removed but not reconstructed in either an immediate or delayed manner. Over more than a decade of practice for some of the authors, we have not had any patient complain of masticatory oscillopsia. We routinely place a pressure patch for three days to displace the orbital contents temporally and keep the temporalis muscle and fat pad out of the orbit and have not encountered significant sequelae from lack of lateral orbital bony support.

In the cases presented herein, we were able to successfully decompress the orbits by resecting the lateral orbital walls, portions of the orbital floors, and portions of the medial orbital wall, through a single transconjunctival incision with extension into a lateral canthotomy. Using preoperative planning, we were able to customize the surgery on each side and balance pre-existing asymmetry, tailor resections to local variations in patient anatomy (position of the anterior cranial fossa, ethmoid air cells, maxillary sinus), with decreased operating time compared with standard technique (75-90 minutes versus $120-150$ minutes). In the future, planning and postoperative assessment will be useful in studying various approaches to decompression to better define accurate, and less surgeon-dependent, algorithms for decompression.

\section{References}

1 Stan MN, Garrity JA, Bahn RS. The evaluation and treatment of graves ophthalmopathy. Med Clin North Am 2012;96(2):311-328

2 Bahn RS. Graves' ophthalmopathy. N Engl J Med 2010;362(8): 726-738
3 Khoo TK, Bahn RS. Pathogenesis of Graves' ophthalmopathy: the role of autoantibodies. Thyroid 2007;17(10):1013-1018

4 Lindholm J, Laurberg P. Hyperthyroidism, exophthalmos, and goiter: historical notes on the orbitopathy. Thyroid 2010;20(3): 291-300

5 Garrity JA, Bahn RS. Pathogenesis of graves ophthalmopathy: implications for prediction, prevention, and treatment. Am J Ophthalmol 2006;142(1):147-153

6 Bahn RS. TSH receptor expression in orbital tissue and its role in the pathogenesis of Graves' ophthalmopathy. J Endocrinol Invest 2004;27(3):216-220

7 Prabhakar BS, Bahn RS, Smith TJ. Current perspective on the pathogenesis of Graves' disease and ophthalmopathy. Endocr Rev 2003;24(6):802-835

8 Verity DH, Rose GE. Acute thyroid eye disease (TED): principles of medical and surgical management. Eye (Lond) 2013;27(3):308-319

9 Boboridis KG, Bunce C. Surgical orbital decompression for thyroid eye disease. Cochrane Database Syst Rev 2011;(12):CD007630

10 Borumandi F, Hammer B, Kamer L, von Arx G. How predictable is exophthalmos reduction in Graves' orbitopathy? A review of the literature. Br J Ophthalmol 2011;95(12):1625-1630

11 Baldeschi L. Small versus coronal incision orbital decompression in Graves' orbitopathy. Orbit 2010;29(4):177-182

12 Leong SC, White PS. Outcomes following surgical decompression for dysthyroid orbitopathy (Graves' disease). Curr Opin Otolaryngol Head Neck Surg 2010;18(1):37-43

13 Borumandi F, Hammer B, Noser H, Kamer L. Classification of orbital morphology for decompression surgery in Graves' orbitopathy: two-dimensional versus three-dimensional orbital parameters. Br J Ophthalmol 2013;97(5):659-662

14 Cascone P, Rinna C, Reale G, Calvani F, Iannetti G. Compression and stretching in Graves orbitopathy: emergency orbital decompression techniques. J Craniofac Surg 2012;23(5):1430-1433

15 Kamer L, Noser H, Kirsch E, Hammer B. Anatomy-based surgical concepts for individualized orbital decompression surgery in graves orbitopathy. II. Orbital rim position and angulation. Ophthal Plast Reconstr Surg 2012;28(4):251-255

16 Hierl T, Krause M, Kruber D, Hümpfner-Hierl H. Positioning of bone segments during navigated surgery. J Oral Maxillofac Surg 2013; 71(2):376-381

17 Lee H, Lee Y, Ha S, Park M, Baek S. Measurement of width and distance of the posterior border of the deep lateral orbital wall using computed tomography. J Craniomaxillofac Surg 2011;39(8): 606-609

18 Tavassol F, Kokemüller H, Müller-Tavassol C, Schramm A, Rücker $\mathrm{M}$, Gellrich NC. A quantitative approach to orbital decompression in Graves' disease using computer-assisted surgery: a compilation of different techniques and introduction of the "temporal cage". J Oral Maxillofac Surg 2012;70(5):1152-1160

19 Shen Y, Paskowitz D, Merbs SL, Grant MP. Retrocaruncular approach for the repair of medial orbital wall fractures: an anatomical and clinical study. Craniomaxillofac Trauma Reconstr 201310.1055/s-0034-1375168

20 Morris DE, Liliav B, Cohen MN. Transcaruncular approach to the isolated medial orbital wall fracture: technical perspective and cadaveric dissection. J Craniofac Surg 2014;25(3):1047-1049

21 Gordon CR, Susarla SM, Yaremchuk MJ. Quantitative assessment of medial orbit fracture repair using computer-designed anatomical plates. Plast Reconstr Surg 2012;130(5):698e-705e 